\title{
Numerical Solution for Accelerated Rotating Disk in a Viscous Fluid
}

\author{
Sajjad Hussain $^{1}$, Farooq Ahmad ${ }^{2 *}$, Mohammad Shafique ${ }^{3}$, Sifat Hussain ${ }^{1}$ \\ ${ }^{1}$ Centre for Advanced Studies in Pure and Applied Mathematics, Bahauddin Zakariya University, Multan, Pakistan \\ ${ }^{2}$ Punjab Higher Education Department, Govt. Degree College Darya Khan, Bhakkar, Pakistan \\ ${ }^{3}$ Department of Mathematics, Gomal University, Dera Ismail Khan, Pakistan \\ Email: sajjad_h96@yahoo.com, farooqgujar@gmail.com,mshafique6161@yahoo.com, siffat2002@gmail.com
}

Received September 23, 2012; revised February 24, 2013; accepted March 2, 2013

Copyright (C) 2013 Sajjad Hussain et al. This is an open access article distributed under the Creative Commons Attribution License, which permits unrestricted use, distribution, and reproduction in any medium, provided the original work is properly cited.

\begin{abstract}
The problem of a disk rotating in a viscous fluid has been investigated. The disk is accelerated with angular velocity proportional to time. Employing suitable similarity transformations the governing partial differential equations are transformed in to ordinary differential form. The resulting equations are solved numerically using SOR method and Simpson's (1/3) rule. The results have been improved by using Richardson's extrapolation. The effect of the non-dimensional parameter s which measures unsteadiness is observed on velocity components, skin friction coefficient and torque of the disk.
\end{abstract}

Keywords: Newtonian Fluids; Numerical Analysis; Rotating Disk

\section{Introduction}

The flow of an incompressible viscous fluid past an infinitely rotating disk was first studied by Von Karman [1] who reduced the necessary Navier-Stokes equations to self-similar form by means of some transformations and derived approximate solutions. Different physical situations were studied in this area by Dolidge [2], Sparrow \& Gregg [3] and Benton [4]. Watson et al. [5] considered the flow past a decelerating porous rotating disk and they studied the effects of time dependent suction or injection velocity on the flow of a viscous fluid. Watson et al. [6] considered the two dimensional channel flow symmetrically driven by accelerating walls. Ariel [7] studied the problem of steady laminar flow of a second grade fluid near a rotating disk. The motion of an electrically conducting fluid film squeezed between two parallel disks in the presence of a transverse magnetic field was studied by Hamza [8]. MHD flow due to non coaxial rotations of an accelerated disk and a fluid at infinity was analyzed by Asghar et al. [9].

Unsteady flows are of importance from the practical point of view and full unsteady Navier-Stokes equations with all the unsteady, nonlinear and viscous terms are difficult to solve whereas exact solutions are rare. However, similarity solution to the governing equations is of

\footnotetext{
"Corresponding author.
}

special interest in case of fluid flow along a rotating disk. Wang [10] studied a viscous fluid between two parallel plates, which are being squeezed or separated with normal velocity proportional to $(1-\alpha t)^{-1 / 2}$ and found similarity solutions of the unsteady Navier-Stocks equations. Ishizawa [11] derived a similarity solution to the case of the unsteady laminar flow between two parallel disks. Pop [12] investigated the problem of unsteady flow past a wall which starts impulsively to stretch from rest. Nazar et al. [13] investigated unsteady boundary layer flow due to a rotating fluid. Xu et al. [14] considered unsteady three dimensional MHD flow and heat transfer in boundary layer over an impulsively stretching plate.

Watson and Wang [15] solved the problem for decaleration of a rotating disk in a viscous fluid for the range $-20 \leq s \leq 0$. They also viewed that similarity solution do not exist for $s>0$ which corresponds to the accelerated rotating disk. In the present work, the numerical solutions have been found for an accelerated $(s \geq 0)$ rotating disk in a viscous fluid. The case $s=0$ corresponds to steady rotation of a disk in a fluid.

\section{Mathematical Analysis}

The fluid flow is unsteady and incompressible. $u, v, w$ are velocity components in cylindrical polar coordinates $(r, \theta$, 
$z$ ). The $z$-axis is the axis of rotation of the disk, with $z=0$ on the surface of the disk. The following similarity transformations are used:

$$
\begin{aligned}
& u=\frac{\Omega_{0} r}{(1-\alpha t)} f^{\prime}(\eta), v=\frac{\Omega_{0} r}{(1-\alpha t)} g(\eta), \\
& w=\frac{-2\left(\nu \Omega_{0}\right)^{1 / 2}}{(1-\alpha t)^{1 / 2}} f(\eta) \text { and } p=\frac{-\rho v \Omega_{0}}{(1-\alpha t)} P(\eta),
\end{aligned}
$$

where $\eta=\left(\frac{\Omega_{0}}{v}\right)^{1 / 2} \frac{z}{(1-\alpha t)^{1 / 2}}$ is the dimensionless variable, $\alpha$ is non dimensional constant and $\Omega_{0}$ is a positive constant. When $\alpha=0$, the problem reduces to the case of the steady rotation of a disk in a fluid. We shall study the case when $\alpha \geq 0$. By using Equation (1), Navier-Stokes equations reduce to a set of nonlinear ordinary differential equations

$$
\begin{aligned}
& f^{\prime \prime \prime}+2 f^{\prime \prime} f+g^{2}-f^{\prime} f^{\prime}=s\left(\frac{1}{2} \eta f^{\prime \prime}+f^{\prime}\right), \\
& g^{\prime \prime}+2 f g^{\prime}-2 f^{\prime} g=s\left(\frac{1}{2} \eta g^{\prime}+g\right), \\
& P^{\prime}=2 f^{\prime \prime}+4 f f^{\prime}-s\left(\eta f^{\prime}+f\right) .
\end{aligned}
$$

where prime denote differentiation with respect to $\eta$ and $s=\alpha / \Omega_{0}$ is non-dimensional parameter which measures unsteadiness.

The associated boundary conditions are

$$
\begin{gathered}
\eta=0: f=0, f^{\prime}=0, g=1, P=P_{0} \\
\eta \rightarrow \infty: f^{\prime}=0, g=0, \\
\text { Let } f^{\prime}=q
\end{gathered}
$$

The Equations (2) and (3) become:

$$
\begin{gathered}
q^{\prime \prime}+2 q^{\prime} f+g^{2}-q^{2}=s\left(\frac{1}{2} \eta q^{\prime}+q\right), \\
g^{\prime \prime}+2 f g^{\prime}-2 q g=s\left(\frac{1}{2} \eta g^{\prime}+g\right)
\end{gathered}
$$

The boundary conditions (5) take the form:

$$
\begin{aligned}
& \eta=0: f=0, q=0, g=1 \\
& \eta \rightarrow \infty: q=0, g=0
\end{aligned}
$$

In order to obtain the numerical solutions of nonlinear ordinary differential Equations (7) and (8), we approximate these equations by central difference approximation at a typical point $\eta=\eta_{n}$ of the interval $[0, \infty)$, we obtain

$$
\begin{aligned}
& \left(1-\left(\frac{h}{4} s \eta-h f_{n}\right)\right) q_{n+1}-\left(2+h^{2}\left(s+q_{n}\right)\right) q_{n} \\
& +\left(1+\left(\frac{h}{4} s \eta-h f_{n}\right)\right) q_{n-1}+h^{2} g_{n}^{2}=0
\end{aligned}
$$

$$
\begin{aligned}
& \left(1-\left(\frac{h}{4} s \eta-h f_{n}\right)\right) g_{n+1}-\left(2+h^{2}\left(s+q_{n}\right)\right) g_{n} \\
& +\left(1+\left(\frac{h}{4} s \eta-h f_{n}\right)\right) g_{n-1}=0
\end{aligned}
$$

where $h$ denotes a grid size and Equation (6) is integrated numerically. Also, the symbols used denote $f_{n}=f\left(\eta_{n}\right), g_{n}=g\left(\eta_{n}\right)$ and $q_{n}=q\left(\eta_{n}\right)$. For computational purpose, we shall replace the interval $[0, \infty)$ by $[0, \beta]$ where $\beta$ is a sufficiently large.

\section{Computational Procedure}

Finite difference Equations (10) and (11) and the first order ordinary differential Equation (6) are solved simultaneously by using SOR method, Smith [16] and Simpson's $(1 / 3)$ rule, Gerald [17] with the formula given in Milne [18] respectively subject to the appropriate boundary conditions.

The order of the sequence of iterations is as follows:

1) The Equations (10) and (11) for the solution of $q$ and $g$ are solved subject to the following boundary conditions:

$$
\begin{aligned}
& q=0, g=1 \text { when } \eta=0 ; \\
& q=0, g=0 \text { when } \eta \rightarrow \infty .
\end{aligned}
$$

2) For the solution of $f$, we use the computed values q from above step in to Equation (6) and integrate by Simpson's (1/3) rule subject to the following initial conditions:

$$
f=0 \text { when } \eta=0 .
$$

3) The optimum value of the relaxation parameter $\omega_{\text {opt }}$ is estimated, to accelerate the convergence of the SOR method.

4) The SOR procedure is terminated when the following criterion is satisfied for each of $q$ and $g$ :

$$
\max \left|U_{i}^{n+1}-U_{i}^{n}\right|<10^{-6}
$$

where $n$ denotes the number of iterations and $U$ stands for each of $q$ and $g$.

The above steps 1 to 4 are repeated for higher grid levels $h / 2$ and $h / 4$. The SOR procedure gives the solution of $f^{\prime}$ and $g$ of order of accuracy $O\left(h^{2}\right)$ due to second order finite differences used for finite-difference equations. While, the Simpson's (1/3) rule gives the order of accuracy $O\left(h^{5}\right)$ in the solution of $f$. Higher order accuracy $O\left(h^{6}\right)$ on the basis of above solutions is achieved by using Richardson's extrapolation, Burden [19].

\section{Numerical Results and Discussion}

The numerical results have been found for the values of 
parameter $s$ for range $0 \leq s \leq 3.0$. In order to check the accuracy of the results, calculation have been made on three different grid sizes namely $h=0.05,0.025$ and 0.0125 . The results for skin friction coefficient $f^{\prime \prime}(0)$ and torque $g^{\prime}(0)$ are presented in Table 1. It is observed from the values given in the Table No. 1 that skin friction coefficient $f^{\prime \prime}(0)$ decreases with increasing values of $s$.

Also, the torque experienced by the disk $g^{\prime}(0)$ becomes more negative with increasing values of $s>0$. It means that rotating disk experiences increasing resistance with increasing values of $s$.

It is imperative to describe the effect of the parameter $s$ on the flow pattern. So the graphical results of the velocity components have been exhibited in the Figures
1-3. Figure 1 shows the axial velocity distribution $f(\eta)$. Figure 2 elucidates the circumferential velocity distribution $g(\eta)$. The radial velocity distribution $f^{\prime}(\eta)$ has been depicted in Figure 3. It is observed that all the velocity components decrease by increasing $s$.

The torque $T$ experienced by the disk of radius $R$, may

Table 1. Data of skin friction coefficient \& torque.

\begin{tabular}{ccc}
\hline$s$ & $f^{\prime \prime}(0)$ & $g^{\prime}(0)$ \\
\hline 0.5 & 0.357811 & -0.785213 \\
1.0 & 0.258781 & -0.948995 \\
1.5 & 0.184577 & -1.101076 \\
2.0 & 0.136933 & -1.238956 \\
2.5 & 0.105831 & -1.364260 \\
3.0 & 0.090728 & -1.435172 \\
\hline
\end{tabular}

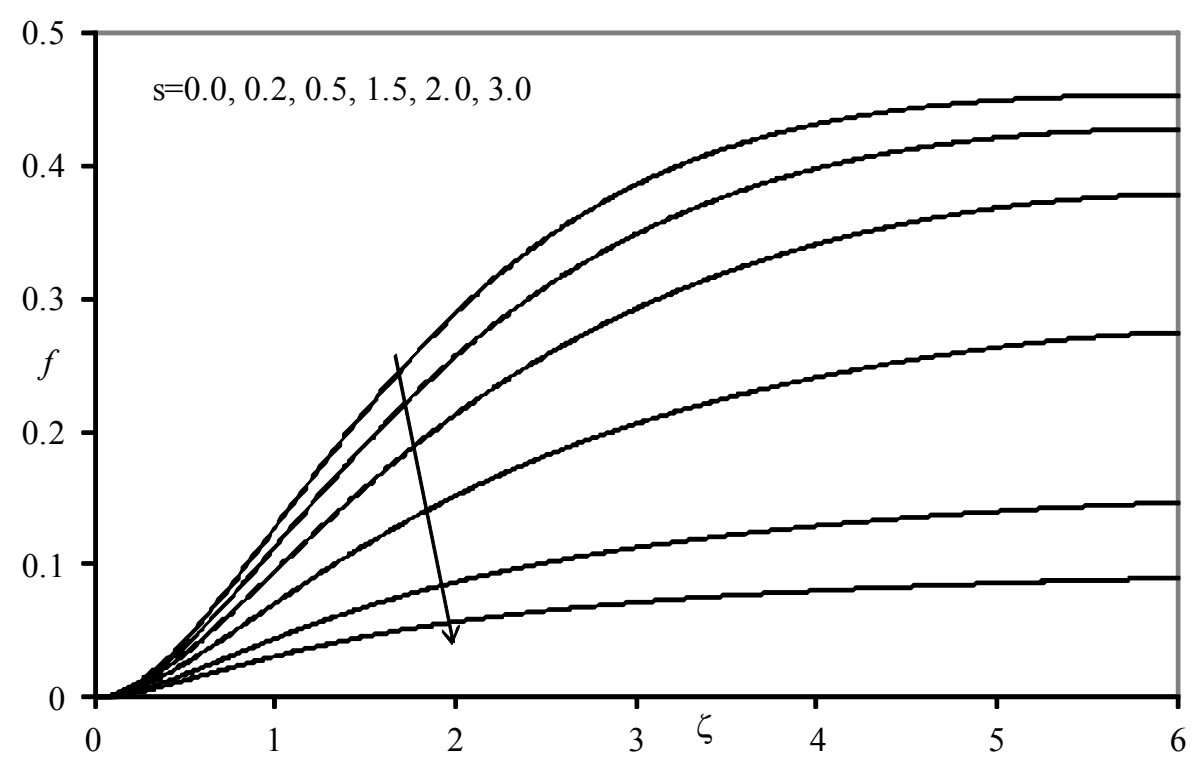

Figure 1. Graph of the axial velocity distribution $\boldsymbol{f}(\boldsymbol{\eta})$ for different values of $s$.

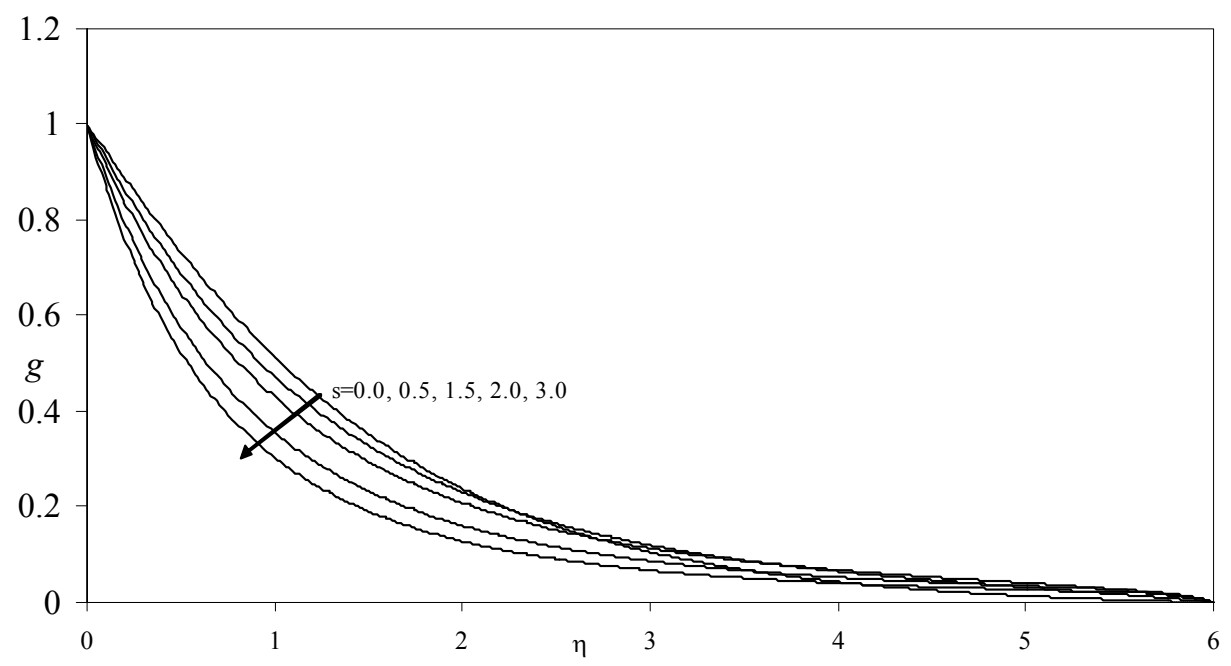

Figure 2. Graph of the circumferential velocity $g(\eta)$ for different values of $s$. 


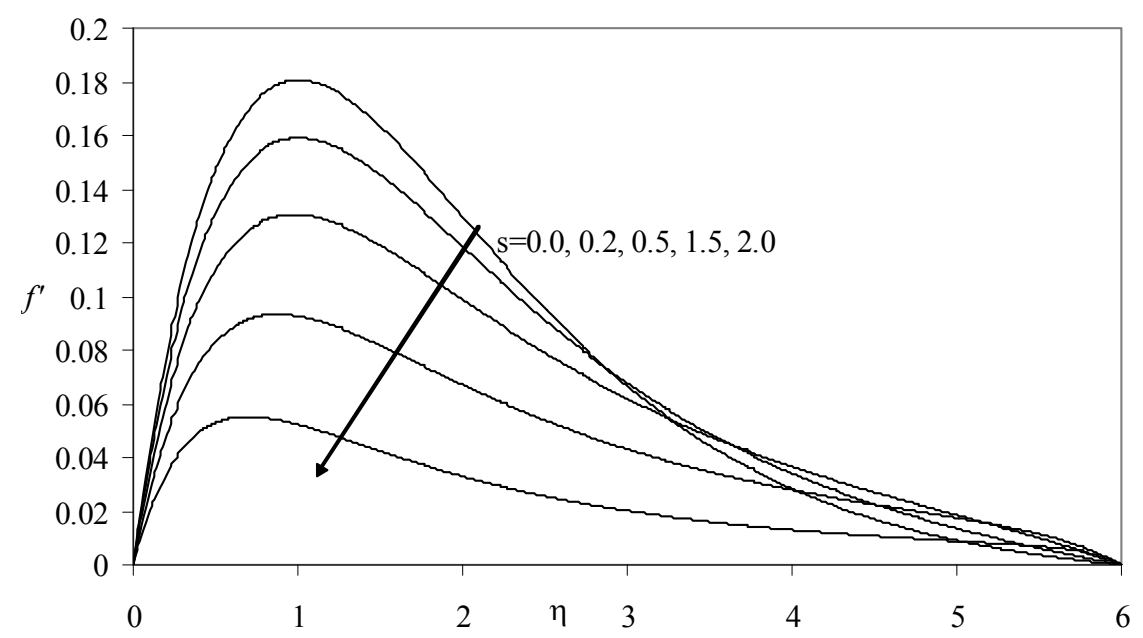

Figure 3. Graph of radial velocity distribution $f^{\prime}(\eta)$ for different values of $s$.

be written as:

$$
\begin{aligned}
& T=\left.2 \pi \rho v \int_{0}^{R} r^{2} \frac{\partial v}{\partial z}\right|_{z=0} \mathrm{~d} r \\
& T=\frac{1}{2} \pi \rho \Omega_{0} \sqrt{v \Omega_{0}} R^{4}(1-\alpha s t)^{-3 / 2} g^{\prime}(0)
\end{aligned}
$$

For positive values of $s$, the rotating disk experiences a resistance, hence $g^{\prime}(0)$ is negative as shown in Table 1.

\section{REFERENCES}

[1] T. Von Karaman, "Uberlaminare und Turbulent Reibung," Zeitschrift für Angewandte Mathematik und Mechanik, Vol. 1, 1921, pp. 233-252.

doi:10.1002/zamm.19210010401

[2] D. E. Dolidge, "Unsteady Motion of a Viscous Liquid Produced by a Rotating Disk," Prikladnaya Matematika $i$ Mekhanika, Vol. 18, 1954, pp. 371-378.

[3] E. M. Sparrow and J. L. Gregg, "Flow about an Unsteadily Rotating Disk," Journal of the Aeronautical Sciences, Vol. 27, No. 4, 1960, pp. 252-257.

[4] E. R. Benton, "On the Flow Due to a Rotating Disk," Journal of Fluid Mechanics, Vol. 24, No. 4, 1966, pp. 781-800. doi:10.1017/S0022112066001009

[5] L. T. Watson, K. K. Sanakara and L. C. Mounfield, "Deceleration of a Porous Rotating Disk in a Viscous Fluid," International Journal of Engineering Science, Vol. 23, 1985, pp. 131-152. doi:10.1016/0020-7225(85)90022-9

[6] E. B. Watson, W. H. H. Banks, M. B. Zaturska and P. G. Drazin, "On Transition to Chaos in Two Dimensional Channel Flow Symmetrically Driven by Accelerating Walls," Journal of Fluid Mechanics, Vol. 212, 1990, pp. 451-485. doi:10.1017/S0022112090002051

[7] P. D. Ariel, "Computation of Flow of a Second Grade Fluid near a Rotating Disk," International Journal of Engineering Science, Vol. 23, 1997, pp. 1335-1357. doi:10.1016/S0020-7225(97)87427-7

[8] E. A. Hamza, "Unsteady Flow between Two Disks with
Heat Transfer in the Presence of a Magnetic Field," Journal of Physics D: Applied Physics, Vol. 25, No. 10, 1992, pp. 1425-1431. doi:10.1088/0022-3727/25/10/007

[9] S. Asghar, K. Hanif, T. Hayat and C. M. Khalique, "MHD non Newtonian Flow Due to Non Coaxial Rotations of an Accelerated Disk and a Fluid at Infinity," Communication in Nonlinear Science and Numerical Simulation, Vol. 12, No. 4, 2007, pp. 465-485. doi:10.1016/j.cnsns.2005.04.006

[10] C. Y. Wang, "The Squeezing of a Fluid between Two Plates," Journal of Applied Mechanics, Vol. 43, No. 4, 1976, pp. 579-583. doi:10.1115/1.3423935

[11] S. Ishizawa, "The Unsteady Laminar Flow between Two Parallel Disks with Arbitrarily Varying Gap Width," Bulletin of JSME, Vol. 9, No. 35, 1966, pp. 533-550. doi:10.1299/jsme1958.9.533

[12] I. Pop, "Unsteady Flow past a Stretching Sheet," Mechanics Research Communications, Vol. 23, No. 4, 1996, pp. 413-422. doi:10.1016/0093-6413(96)00040-7

[13] R. Nazar, N. Amin and I. Pop, "Unsteady Boundary Layer Flow Due to a Rotating Fluid," Mechanics Research Communications, Vol. 31, 2004, pp. 121-128. doi:10.1016/j.mechrescom.2003.09.004

[14] H. Xu, S. J. Liao and I. Pop, "Series Solution of Unsteady Three Dimensional MHD Flow and Heat Transfer in Boundary Layer over an Impulsively Stretching Plate," European Journal of Mechanics, Vol. 26, No. 1, 2007, pp. 15-27. doi:10.1016/j.euromechflu.2005.12.003

[15] L. T. Watson and C. Y. Wang, "Deceleration of a Rotating Disk in a Viscous Fluid," Physics of Fluids, Vol. 22, No. 12, 1979, pp. 226-2269. doi:10.1063/1.862535

[16] G. D. Smith, "Numerical Solution of Partial Differential Equation," Clarendon Press, Oxford, 1979.

[17] C. F. Gerald, "Applied Numerical Analysis," AddisonWesley Publication, New York, 1989.

[18] W. E. Milne, "Numerical Solution of Differential Equation," Dover Publication, New York, 1970.

[19] R. L. Burden, "Numerical Analysis," Prindle, Weber \& Schmidt, Boston, 1985. 